\title{
Preparation of silver nanoparticles in a high voltage AC arc in water
}

\author{
Joanna Jabłońska' Krzysztof Jankowski $^{1,2}$ (1) $\cdot$ Mikołaj Tomasik $^{2} \cdot$ Dariusz Cykalewicz $^{2} \cdot$ Paweł Uznański $^{3}$. \\ Szymon Całuch ${ }^{2} \cdot$ Mirosław Szybowicz $^{1} \cdot$ Joanna Zakrzewska ${ }^{3} \cdot$ Paweł Mazurek $^{2}$
}

Received: 2 April 2020 / Accepted: 6 January 2021 / Published online: 1 February 2021

(C) The Author(s) 2021 OPEN

\begin{abstract}
The article presents for the first time the synthesis of silver nanoparticles in an electric arc of high-voltage alternating current with a frequency of $50 \mathrm{~Hz}$. In particular, the method and apparatus necessary for the preparation of nanoparticles in water solution is discussed. Current-voltage characteristics depending on the mutual distance between the electrodes are presented which show a very high stability of the generated discharge phenomena. The obtained nanoparticles were examined using various analytical techniques such as UV-Vis spectroscopy, dynamic light scattering (DLS), zeta potential, energy dispersive X-Ray analysis (EDS), X-ray diffraction (XRD), and X-ray fluorescence (EDXRF). The morphology, surface and size of the obtained nanoparticles was carried out using transmission electron microscopy (TEM) and scanning TEM (STEM) equipped with the annual dark-field imaging scanning atomic-scale chemical mapping (STEM). The designed simple power supply unit consisting of an autotransformer and a microwave oven transformer (MOT) makes the preparation of silver nanoparticles both simple and economical.
\end{abstract}

Keywords Silver nanoparticles synthesis · Submerged arc discharge method · High voltage AC arc discharge ·

Alternating current

\section{Introduction}

Due to their biocidal, antibacterial and antiviral properties, silver nanoparticles are widely used in medicine and health care [1-4], although the applications in other fields of science and technology, such as in chemical analysis (SERS) [5-7] and electronics [8] are also very significant. Their specific uses strictly depend on the method of synthesis which include: inert gas condensation [9-14], radiolysis [15-17], sol-gel method [18-20] ion implantation [21], chemical vapour deposition (CVD) [22], polymerization $[23,24]$ or synthesis by chemical reduction from silver salts and organometallic precursors [25]. These commonly known methods belong to bottom-up approach of nanoparticles synthesis. The submerged arc discharge synthesis method described here, together with e.g. laser ablation
$[26,27]$, belongs in turn to the top-down methodology in nanoscience and is considered both the simplest and the cheapest. It consists in creating an arc discharge between the silver electrodes immersed in a liquid medium. The generated high temperature associated with the shortcircuit current flow leads to ablation of the bulk material of the electrodes with a formation of small metal objects [28-32]. At present, most solutions for generating an arc between electrodes are based on the use of direct current [33]. Unfortunately, such a solution is associated with a quite expensive electric current rectification system to maintain the stability of the power supply. In this communication, instead of a low-voltage rectifier supply, we propose a solution based on the generation of a high-voltage arc in which the synthesis of silver nanoparticles also takes place. We observed that this solution accelerates the

\footnotetext{
$\triangle$ Krzysztof Jankowski, kjankowski@ajp.edu.pl | ${ }^{1}$ Faculty of Materials Engineering and Technical Physics, Poznan University of Technology, Piotrowo 3A, 61-138 Poznań, Poland. ${ }^{2}$ Technical Department, The Jacob of Paradies University, Chopina 52, 66-400 Gorzów Wielkopolski, Poland. ${ }^{3}$ Centre of Molecular and Macromolecular Studies, Polish Academy of Sciences, Sienkiewicza 112, 90-363 Lodz, Poland.
} 
process of producing nanoparticles due to the stabilization the electric arc between the electrodes. To the best of our knowledge the presented solution based on MOT transformer seems to be the simplest method of making high voltage $\mathrm{AC}$ arc thanks to appropriate power systems, creates a stable arc between the electrodes and it is powerful, and cost effective.

\section{Materials and method}

\subsection{Materials}

Silver rods with a purity of $\geq 99.99 \%$ and a diameter of $2 \mathrm{~mm}$ were purchased from Mint- Metals Poland and were used as electrodes. The submerged arc process was carried out in a distilled water with a resistivity of $18.2 \mathrm{Mohm} / \mathrm{cm}$.

\subsection{Analysis methods}

The voltage parameters were measured using a DP-100 high-voltage differential probe with the following parameters: accuracy $\pm 2 \%$, the bandwidth of the DC $3 \mathrm{~dB}$ is $100 \mathrm{MHz}$, differential input impedance $54 \mathrm{Mohm}$ with a $3.5 \mathrm{~ns}$ rise time. The current measurement was made using a PA-677 probe with a DC- $1 \mathrm{MHz}$ range up to $70 \mathrm{~A}$. The signals were monitored with a Rigol MSO1074Z oscilloscope.

The absorbance spectrum of the colloidal sample was measured in the range of 320-900 nm, using a UV-Vis spectrometer Shimadzu-UV 1240 with distilled water as a reference. The size distribution and stability of AgNPs were performed by measuring DLS and Zeta potential measurements using Malvern Zetasizer Nano ZS90 instrument. X-ray diffraction (XRD) analysis was conducted on a DRON diffractometer (Russia) using monochromatic $\mathrm{Cu}$ Ka radiation $(\lambda=1.5406 \AA)$ operated at $50 \mathrm{kV}$ and $40 \mathrm{~mA}$ at the accusation scan $\theta-2 \theta$. The scanning was done in the $2 \theta$ range of $5^{\circ}-70^{\circ}$. The morphology, shape and size of the silver nanoparticles were examined by TEM technique with a Talos F200X. Atomic-scale chemical maps and energydispersive X-ray spectra (EDS) were obtained using scanning transmission electron microscopy STEM-EDS/JEOL JEM 2800. Elemental analysis chemical was performed using the EDXRF technique on an Epsilon 1 portable XRF Analyzer from Malvern Pananalytical.

\section{Experimental details}

The process of producing silver nanoparticles takes place in the current arc between two silver electrodes placed opposite each other at an angle of $60^{\circ}$. The electrodes were $50 \mathrm{~mm}$ long and $2 \mathrm{~mm}$ in diameter and were immersed in water to a depth of $2 \mathrm{~cm}$. Figure 1a shows a scheme of glassy reactor for the synthesis of nanoparticles a glassy vessel is filled with distilled water and equipped with a magnetic stirrer for uniform temperature distribution of the water medium and homogeneous distribution of the produced nanoparticles. The high voltage alternating current arc was generated using a power supply (Fig. 1b). It consists of an autotransformer with an adjustable output voltage in the range of $0-230 \mathrm{~V}$, which supplies the highvoltage transformer typically used in microwave ovens, so-called MOT, and it allows the generation of an output voltage between the electrodes in the range from 0 to $2 \mathrm{kV}$. The stepper motor precisely controls the position of the electrodes relative to each other. The measured distance between the electrode tips optimal for generating the electric arc of the discharge was $1.2 \mathrm{~mm}$.

In the experiments, the output voltage of the autotransformer was set at $100 \mathrm{~V}$, which generated a voltage of $1100 \mathrm{~V}$ on the secondary winding of the high voltage transformer (MOT). The process of synthesis of silver particles was carried out in the electric arc in two time regimes-for 5 min (sample A) and 20 min (sample B). The temperature of the $250 \mathrm{~mL}$ water medium was not stabilized during the arc. Thereby it rose in the reaction vessel from $19{ }^{\circ} \mathrm{C}$ before the process to 32.5 and $63^{\circ} \mathrm{C}$ during the 5 and $20 \mathrm{~min}$ arc, respectively. The loss of weight of the electrodes is not uniform and was $0.0013 \mathrm{mg}$ for the first one and $0.0011 \mathrm{mg}$ for the second in a $5 \mathrm{~min}$ process. For a $20 \mathrm{~min}$ arc, the weight loss of the electrodes was $0.0044 \mathrm{~g}$ and $0.0033 \mathrm{~g}$, respectively. Thus the overall weight loss of the silver electrodes was $0.0024 \mathrm{~g}$ and $0.0077 \mathrm{~g}$ for the arc durations used. From the first mass of silver, $5.610^{14}$ spherical particles with a diameter of $92 \mathrm{~nm}$ can be obtained and from the second one $1.8010^{15} \mathrm{NPs}$.

\section{Results and discussion}

\subsection{Current-voltage characteristics}

Figure 2 shows the current-voltage waveforms during NPs synthesis in an AC arc. The open circuit voltage between the silver electrodes before starting the process was $1.1 \mathrm{kV}$ AC. During the process, the maximum amplitude of the voltage between the electrodes decreases to $850 \mathrm{~V}(1700 \mathrm{~V}$ peak-to-peak voltage), and maximum current was set to 0.8 A (1.6 A peak-to-peak current).

\subsection{UV-Vis spectral analysis}

The Ag nanoparticles concentration was controlled with the arc discharge time, which was 5 and $20 \mathrm{~min}$. The formation of the Ag NPs during the HV AC process was 
Fig. 1 Scheme of the apparatus for the synthesis of AgNPs in arc discharge: a electrode guide system: 1 . arc discharge zone, 2. silver electrodes, 3. electrodes holder, 4. steeper motor, 5 . glass vessel, 6 . DI (deionized water), 7. Magnetic stirrer. $\mathbf{b}$ General scheme of power supply
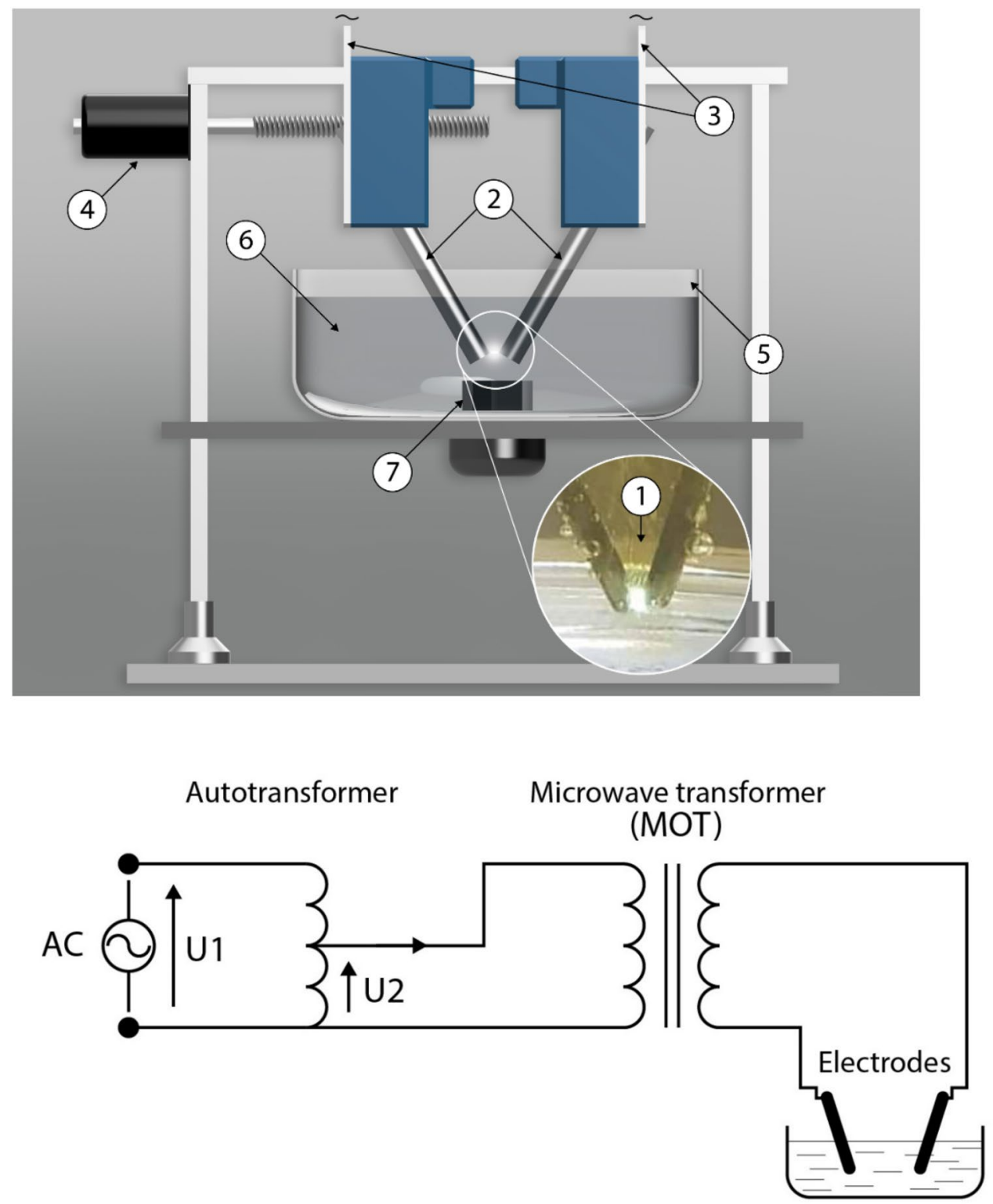

followed by change in the colour of the reaction solution from colourless by yellow to dark orange, as illustrate photographs in Fig. 3. The UV-Vis absorption spectra of the Ag NPs with different concentrations are shown in Fig. 4. The broad band with a maximum at $404 \mathrm{~nm}$ (sample $A$ ) derives from the phenomenon of surface plasmon resonance characteristic of silver nanoparticles [33]. The profile of the band with long absorption tail suggest the presence of not only spherical nanoparticles in the water but objects of various shapes. For sample $B$, the spectrum position slightly shifts to $406 \mathrm{~nm}$ and widens at $\sim 500 \mathrm{~nm}$, indicating a minor change in the size distribution of the NPs.

\subsection{Dynamic light scattering (DLS) and zeta potential results}

The size distribution profile of the synthesized silver nanoparticles measured by the DLS method is shown in Fig. 5. The scattered signal confirms the broad particle size distribution which agrees well with the unsymmetrical extinction peak in the long wavelength absorption region observed in the UV-Vis spectra, especially for sample B. Size distribution profiles reveal two populations of NPs for each sample with the average size around of 18 and $90 \mathrm{~nm}$. The shorter synthesized colloid A shows a narrower size distribution. Also the zeta 
Fig. 2 Current-voltage characteristics during nanoparticles synthesis as a function of time
Fig. 3 Silver nanoparticles colloids obtained after 5 (Sample A) and 20 min (sample B) of the AC discharge process
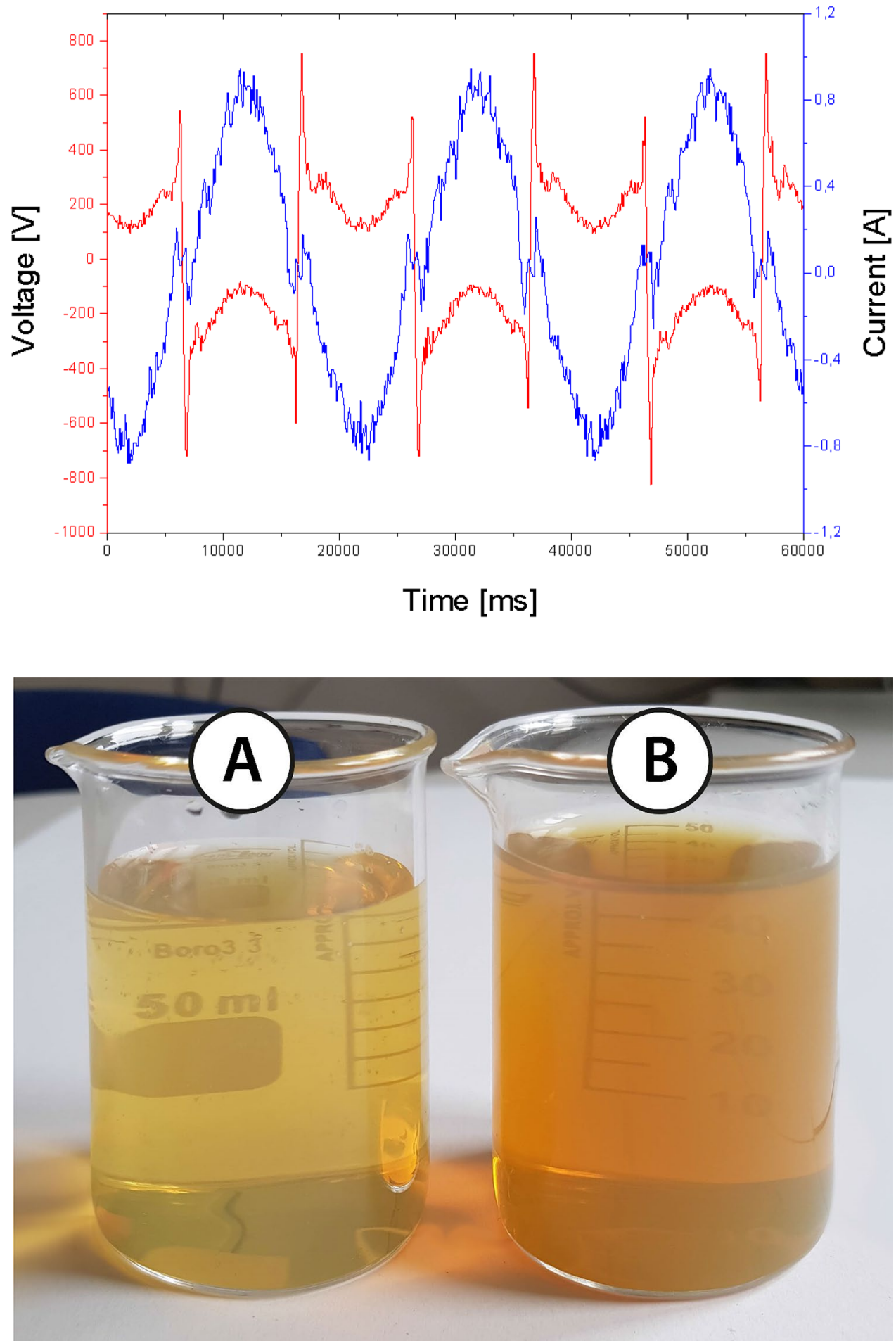

potential distributions have a similar position for both colloids: at -20.4 for sample $A$, and $-22.31 \mathrm{mV}$ for sample B (Fig. 6). The calculated average particle size is $48.9 \mathrm{~nm}$ and 46.8 for a $5 \mathrm{~min}$ and 20 min sample, respectively. In turn, the zeta potential of the synthesized Ag NPs is a sharp peak at -20.4 for sample $A$, and $-22.31 \mathrm{mV}$ for sample B (Fig. 6), but for the last one it is wider. These values suggests that the surface of the silver nanoparticles dispersed in water medium is positively charged. High negative values are responsible for the repulsion between the particles and make the colloid very stable for at least months. 


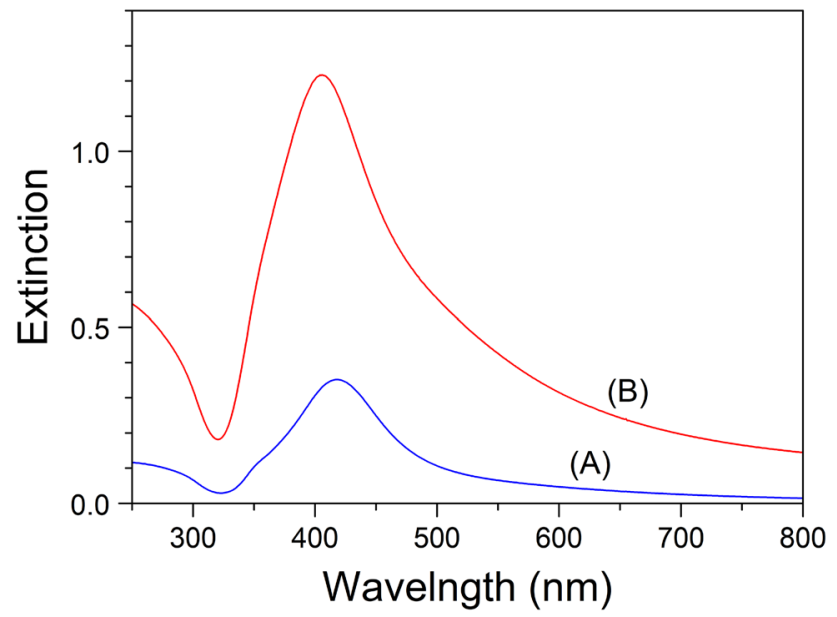

Fig. 4 UV-Vis spectra of synthesized Ag NPs at various concentrations obtained during a 5-min (a) and 20-min (b) process in the HV AC arc measured in $1 \mathrm{~mm}$ path length cuvette. $(404 \mathrm{~nm}),(406 \mathrm{~nm})$

\subsection{Transmission electron microscope (TEM)}

TEM images of the colloidal silver nanoparticles obtained in the HV AC arc discharge confirm their heterogeneity. Interestingly, they also show the lack of aggregates or large particles observed in syntheses by other methods in the current arc [34]. Although nanoparticles are mostly oval in shape (Fig. 7a), triangular or trapezoidal nanoparticles are also clearly visible (Fig. 7b). The measured particles size varies from 20 to $100 \mathrm{~nm}$, but their shape is not related to a specific size. Interestingly, TEM photographs taken at higher magnifications reveal a $4 \mathrm{~nm}$ thick shell around the obtained nanostructures.

\subsection{Chemical maps of Ag NPs on the atomic-scale by high-angle annular dark-field imaging (HAADF) using a scanning transmission electron microscope (STEM) technique}

The atomic composition and morphology of the synthesized NPs as well as the thin coating around NPs visible at higher magnifications (Fig. 7) was examined by the STEM method. Figure 8 presents the HAADF atomic-scale chemical maps. The shape of objects, which are of representative in size, is visualized only in the case of $\mathrm{Ag}, \mathrm{Cu}, \mathrm{C}, \mathrm{Si}$ and $\mathrm{O}$ atoms. The presence of copper atoms is inherently related to the STEM measurement methodology and originates from the reflections of the copper mesh covered with amorphous carbon. The presence of $\mathrm{Si}$ and $\mathrm{O}$ may indicate the composition of the thin layer visible in the TEM image
Fig. 5 Dynamic light scattering size distribution by intensity of synthesized Ag NPs for 5 min (sample A) and for $20 \mathrm{~min}$ (sample B)
(A)

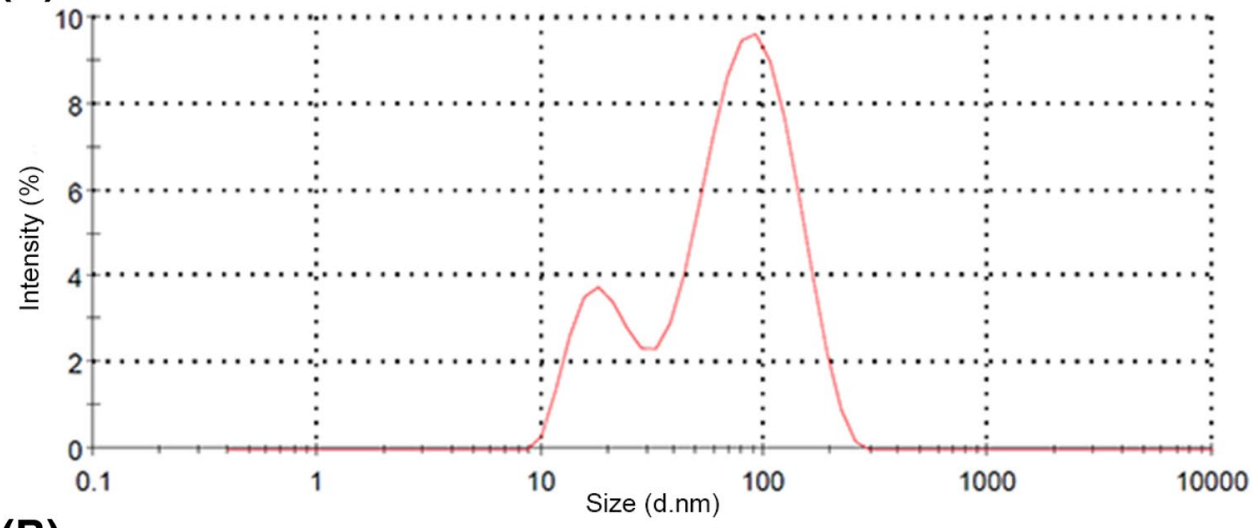

(B)

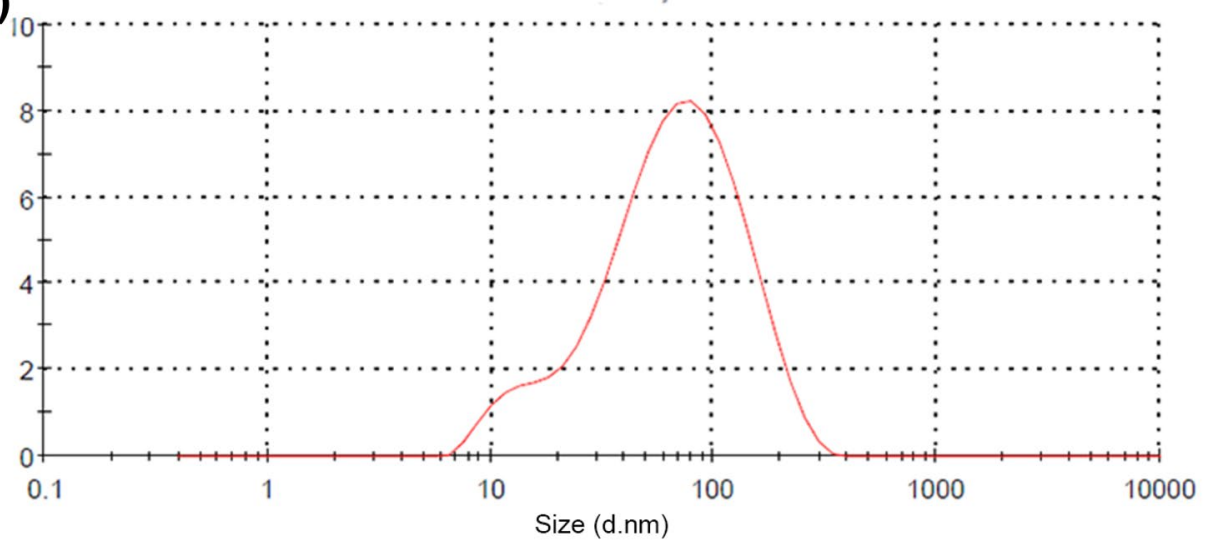

SN Applied Sciences 
Fig. 6 The Zeta potential of AgNPs colloidal solutions of sample $A$ and sample $B$
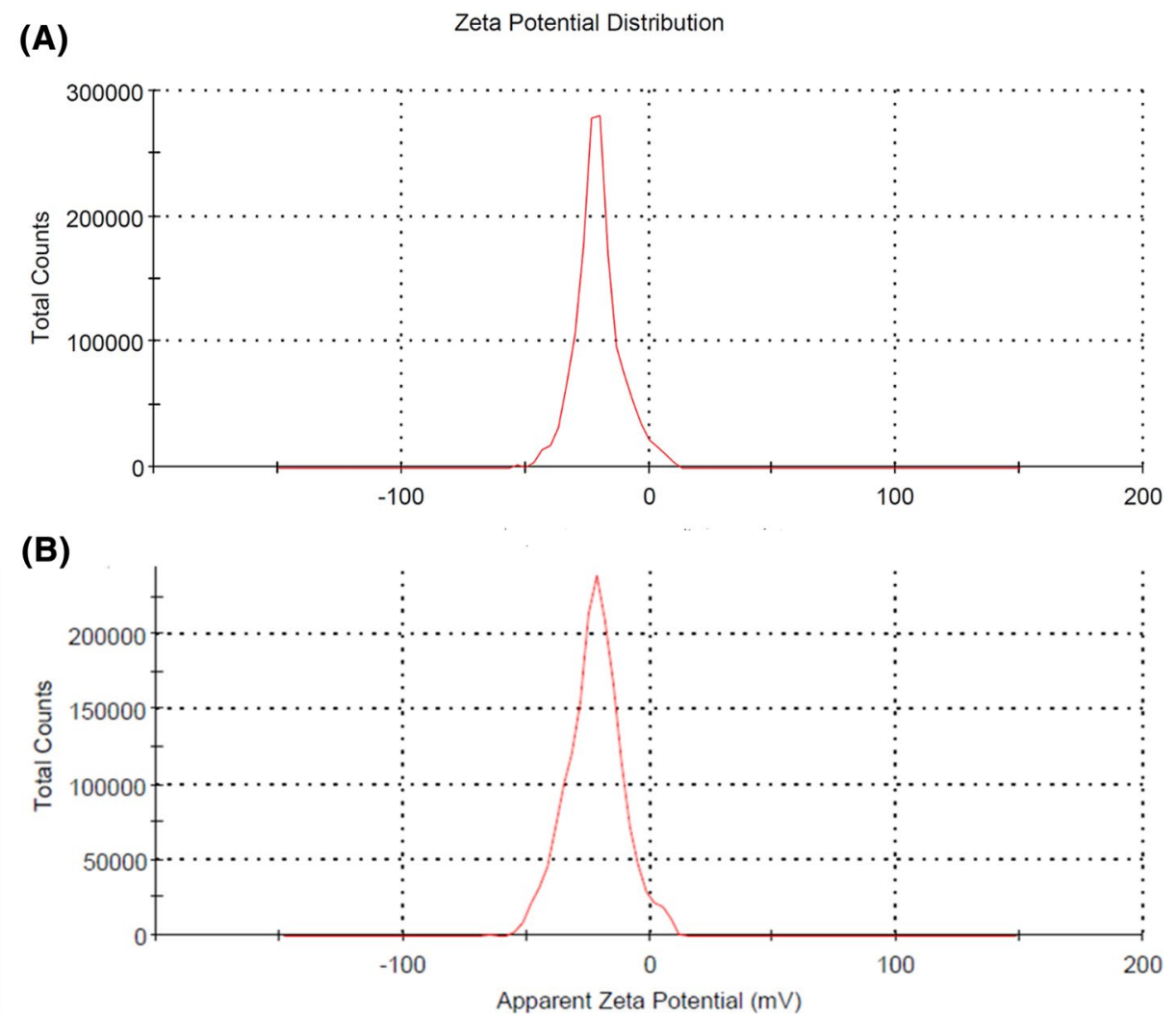

(Fig. 7) and may result from the walls of the glass reactor. During the discharge in $\mathrm{HV}$ alternating current arc, dissociation of water molecules with the formation of reactive $\mathrm{H}^{*}$ and $\mathrm{OH}^{*}$ radicals and dissociation of oxygen molecules dissolved in the aqueous medium with the formation of singlet oxygen may occur. Such species, due to their high reactivity, can be trapped on the Ag surface and finally stabilize the colloid electrostatically. Energy dispersive spectrum of the synthesized Ag nanoparticles also shows the presence of silver (strong signal peak at $3 \mathrm{keV}$ ) as the basic component and cooper at $8 \mathrm{keV}$. The remaining elements such as $\mathrm{O}$ and $\mathrm{Si}$, although were registered qualitatively are at trace level. The carbon atoms are the result of a thin amorphous layer covering the copper mesh.

\subsection{X-Ray fluorescence (XRF) measurements}

The XRF technique has been used complementary to EDS/ STEM to determine elemental composition of the silver colloid. Again, the presence of dominant Ag element is confirmed on XRF spectrum (Fig. 9). The spectrum contains peaks of silver: $\mathrm{K}_{\alpha}$ line at $22.16 \mathrm{keV}$ and $\mathrm{K}_{\beta}$ line at $24.9 \mathrm{keV}$. Also characteristic silver L-line was observed with the $\mathrm{L}$-absorption edge at $3.82 \mathrm{keV}$. In addition to the silver element, Si was also detected in trace amounts.

\subsection{X-Ray diffraction (XRD) Studies}

X-ray diffraction analysis is very important for determination the structure of the nanocrystals and their morphology, as the nanoparticles have been produced in non-equilibrium conditions of very high temperature and ionizing voltage. Figure 10 shows the XRD pattern of the obtained nanoparticles. The diffraction clearly shows the characteristics reflection $2 \theta$ peaks at $38.2,44.4$, and $64.6^{\circ}$ corresponding to the (111), (200), (220) planes of fcc lattice of metallic silver, respectively [35], [36]. The average crystalline size of the silver nanoparticles was estimated using the Debye-Scherrer's equation (Eq. 1) on the basis of determining the width of the peak (111) in Bragg reflection.

$D=\frac{0.9 \lambda}{\beta \cos \theta}$

where $D$ is the mean size of the crystallites, $\lambda$ is the $X$-ray wavelength, $\beta$ is the line broadening at half the maximum intensity (FWHM) in radians, 0.9 is the value of the shape factor and $\theta$ is the Bragg angle. The estimated average size 
Fig. 7 Transmission electron microscopy images showing silver nanoparticles from AC high voltage arc discharge for 5 min (Panel A) and 20 min (Panel B) process taken at various magnifications
(A)

(b)
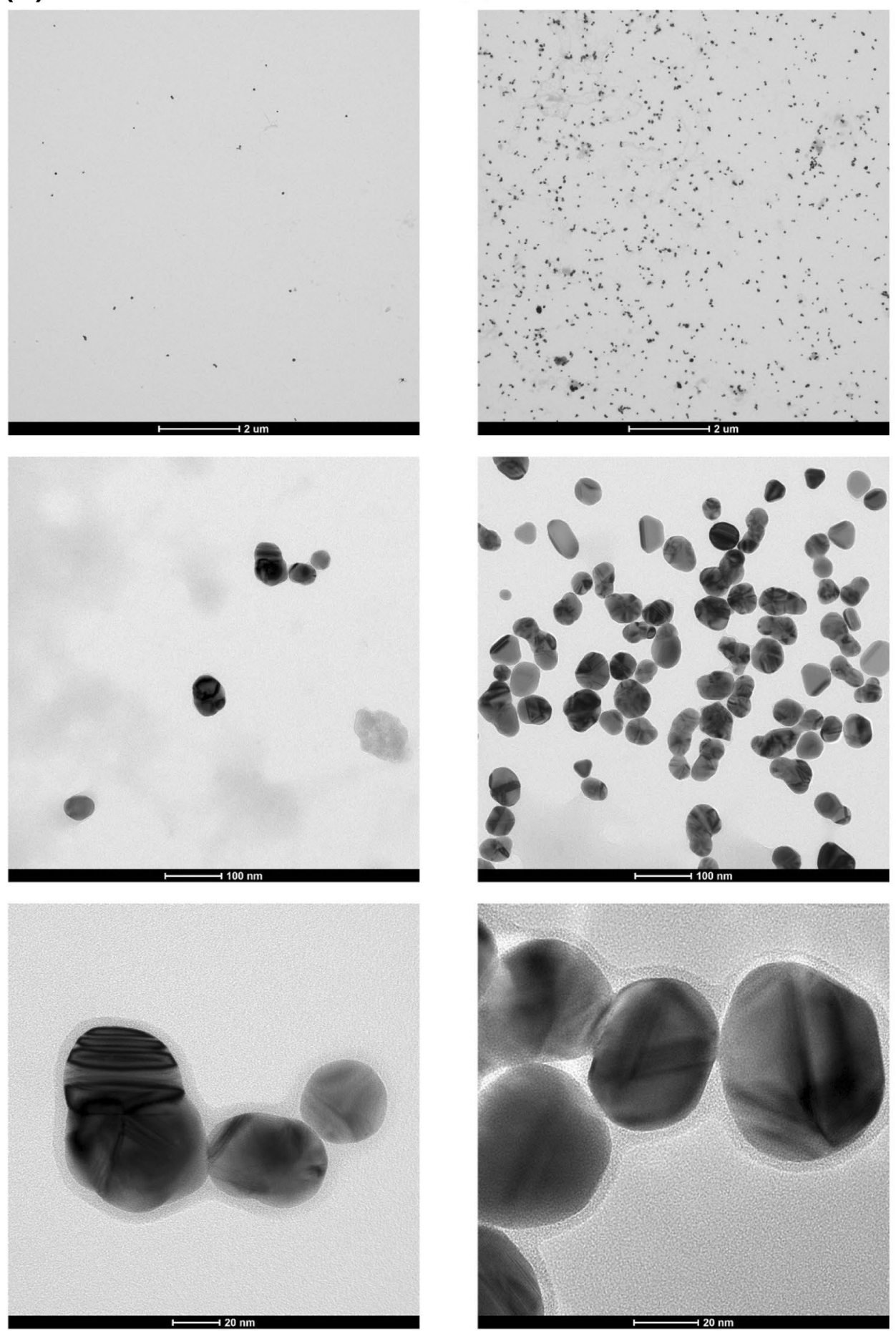

of the silver crystalline domains is $9 \mathrm{~nm}$ and is smaller than the size of nanoparticles observed from TEM images. This means that the nanoparticles growing in the HV discharge arc are made of smaller crystallites.

\section{Summary}

The presented solution consists in omitting the electric current rectification system in the process of electric arc generation for the synthesis of metal particles. Instead, the use of a high-voltage alternating current arc with a frequency of $50 \mathrm{~Hz}$ can be successfully used to produce silver 

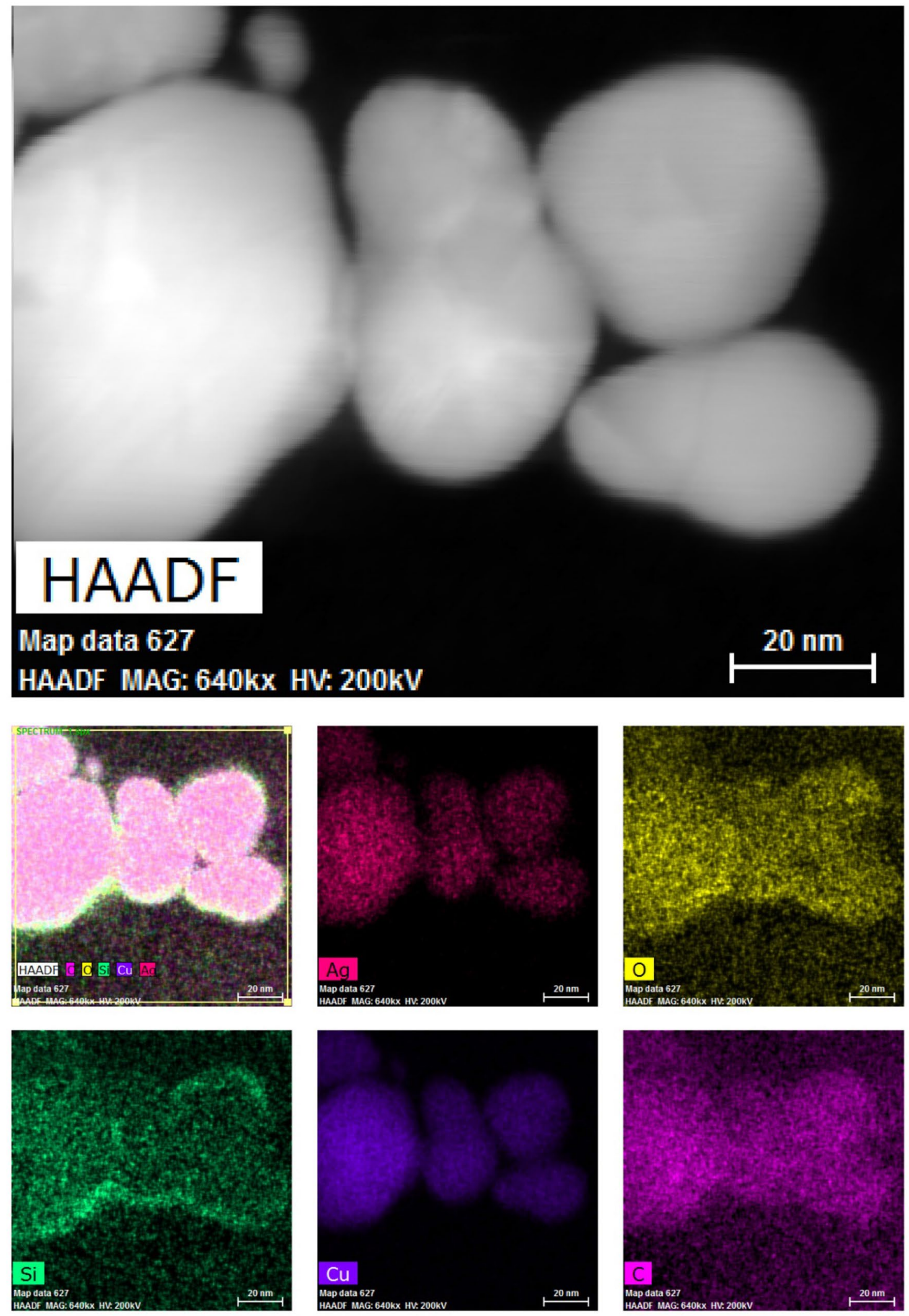

Fig. 8 HAADF/STEM chemical mapping images of silver nanoparticles 
Fig. 9 XRF spectrum of synthesized AgNPs

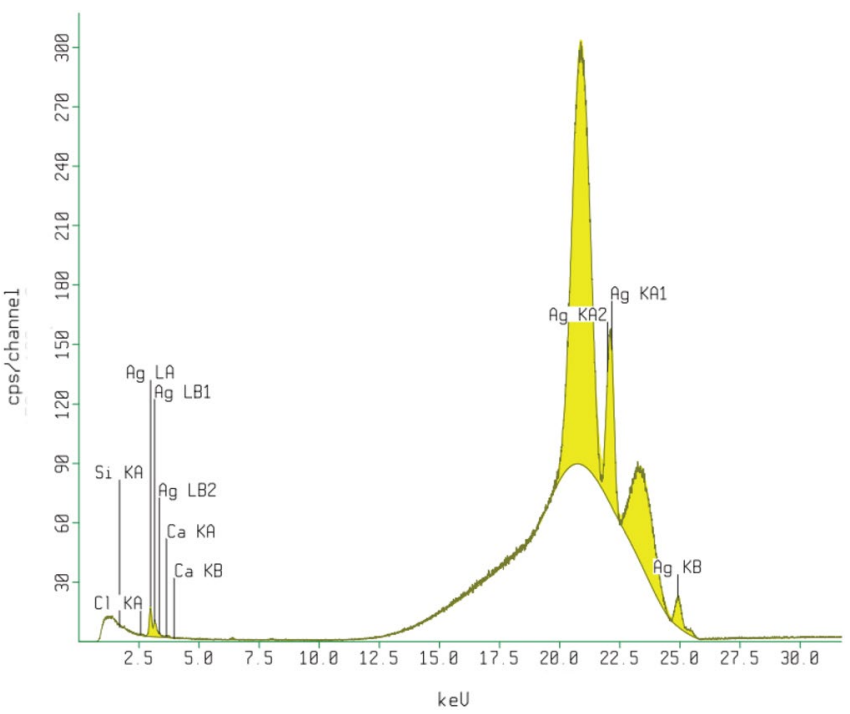

by introducing to the submerged medium various stabilizing agents. The new method of particle synthesis may also apply to other metals and semiconductors from which the electrodes will be made.

Acknowledgements We thank Karina Kalota, BS (JCC Technologies Inc.), Agnes Ostafin, PhD (University of Utah), Elżbieta Skorupska-Raczyńska (Jacob of Paradies University). Acknowledgements The author would like to thank the reviewers for their valuable comments on the paper.

\section{Compliance with ethical standards}

Conflict of interest The authors declares that they have no conflicts of interests.

Open Access This article is licensed under a Creative Commons Attribution 4.0 International License, which permits use, sharing, adaptation, distribution and reproduction in any medium or format, as long as you give appropriate credit to the original author(s) and the source, provide a link to the Creative Commons licence, and indicate if changes were made. The images or other third party material in this article are included in the article's Creative Commons licence, unless indicated otherwise in a credit line to the material. If material is not included in the article's Creative Commons licence and your intended use is not permitted by statutory regulation or exceeds the permitted use, you will need to obtain permission directly from the copyright holder. To view a copy of this licence, visit http://creativecommons .org/licenses/by/4.0/.

\section{References}

1. Minchin R (2008) Sizing up targets with nanoparticles. Nat Nanotech 3:12-13. https://doi.org/10.1038/nnano.2007.433

2. De M, Ghosh PS, Rotello VM (2008) Applications of nanoparticles in biology. Adv Mater 20:4225-4241. https://doi.org/10.1002/ adma.200703183

3. Wilson BC, Patterson MS (2008) The physics, biophysics and technology of photodynamic therapy. Phys Med Biol 53:R61R109. https://doi.org/10.1088/0031-9155/53/9/r01 effect including both electrostatic as well as steric factors 
4. Alexis F, Pridgen EM, Langer R, Farokhzad OC (2010) Nanoparticle technologies for cancer therapy. In: Schäfer-Korting M (ed) Drug delivery. Springer, Berlin, Heidelberg, pp 55-86

5. Zong C, Xu M, Xu L-J, Wei T, Ma X, Zheng X-S, Hu R, Ren B (2018) Surface-enhanced raman spectroscopy for bioanalysis: reliability and challenges. Chem Rev 118:4946-4980. https://doi. org/10.1021/acs.chemrev.7b00668

6. Rycenga M, Cobley CM, Zeng J, Li W, Moran CH, Zhang Q, Qin D, Xia Y (2011) Controlling the synthesis and assembly of silver nanostructures for plasmonic applications. Chem Rev 111:36693712. https://doi.org/10.1021/cr100275d

7. Li W, Camargo PHC, Lu X, Xia Y (2009) Dimers of silver nanospheres: facile synthesis and their use as hot spots for surfaceenhanced raman scattering. Nano Lett 9:485-490. https://doi. org/10.1021/nl803621x

8. Ahn BY, Duoss EB, Motala MJ, Guo X, Park S-I, Xiong Y, Yoon J, Nuzzo RG, Rogers JA, Lewis JA (2009) Omnidirectional printing of flexible, stretchable, and spanning silver microelectrodes. Science 323:1590-1593. https://doi.org/10.1126/science.1168375

9. Granqvist CG, Buhrman RA (1976) Ultrafine metal particles. J Appl Phys 47:2200-2219. https://doi.org/10.1063/1.322870[10] J.Bai,J.-P.Wang,AppliedPhysicsLetters2005,87,152502

10. Bai J, Wang J-P (2005) High-magnetic-moment-core-shell-type FeCo-Au/Ag nanoparticles. Appl Phys Lett 87:152502

11. Hahn H, Averback RS (1990) The production of nanocrystalline powders by magnetron sputtering. J Appl Phys 67:1113-1115. https://doi.org/10.1063/1.345798

12. Remita S, Mostafavi M, Delcourt MO (1996) Bimetallic Ag Pt and $\mathrm{Au} \mathrm{Pt}$ aggregates synthesized by radiolysis. Radiat Phys Chem 47:275-279. https://doi.org/10.1016/0969-806x(94)00172-g

13. Du J-J, Chen C, Gan Y-L, Zhang R-H, Yang C-Y, Zhou X-W (2014) Facile one-pot hydrothermal synthesis of Pt nanoparticles and their electrocatalytic performance. Int J Hydrog Energy 39:17634-17637. https://doi.org/10.1016/j.ijhydene.2014.08.044

14. Marignier JL, Belloni J, Delcourt MO, Chevalier JP (1985) Microaggregates of non-noble metals and bimetallic alloys prepared by radiation-induced reduction. Nature 317:344-345. https:// doi.org/10.1038/317344a0

15. Yeh CL, Kuo CW, Chu YC (2010) Formation of Ti3AIC2/Al2O3 and Ti2AIC/Al2O3 composites by combustion synthesis in TiAl-C-TiO2 systems. J Alloy Compd 494:132-136. https://doi. org/10.1016/j.jallcom.2010.01.043

16. Yan ZX, Deng J, Luo ZM (2010) A comparison study of the agglomeration mechanism of nano- and micrometer aluminum particles. Mater Charact 61:198-205. https://doi.org/10.1016/j. matchar.2009.11.010

17. Khan R, Mohd K, Hameedullah AA, Lohani KR, Ahmad I, Husain FM, Khan W, Alam M (2014) Flower-shaped ZnO nanoparticles synthesized by a novel approach at near-room temperatures with antibacterial and antifungal properties. IJN. https://doi. org/10.2147/ijn.s47351

18. Xiang X, Zu XT, Zhu S, Wang LM (2004) Optical properties of metallic nanoparticles in Ni-ion-implanted a-Al2O3 single crystals. Appl Phys Lett 84:52-54. https://doi.org/10.1063/1.16368 17

19. Adachi M, Tsukui S, Okuyama K (2003) Nanoparticle formation mechanism in CVD reactor with ionization of source vapor. J Nanopart Res 5:31-37. https://doi.org/10.1023/a:1024424518 822

20. Alhareth K, Vauthier C, Gueutin C, Ponchel G, Moussa F (2010) Doxorubicin loading and in vitro release from poly(alkylcyanoacrylate) nanoparticles produced by redox radical emulsion polymerization. J Appl Polym Sci 119:816-822. https://doi.org/10.1002/app.32789

21. Anton N, Benoit J-P, Saulnier P (2008) Design and production of nanoparticles formulated from nano-emulsion templates- $A$ review. J Control Release 128:185-199. https://doi.org/10.1016/j. jconrel.2008.02.007

22. Yin H, Yamamoto T, Wada Y, Yanagida S (2004) Large-scale and size-controlled synthesis of silver nanoparticles under microwave irradiation. Mater Chem Phys 83:66-70. https://doi. org/10.1016/j.matchemphys.2003.09.006

23. Kawakami Y, Seto T, Yoshida T, Ozawa E (2002) Gold nanoparticles and films produced by a laser ablation/gas deposition (LAGD) method. Appl Surf Sci 197-198:587-593. https://doi. org/10.1016/s0169-4332(02)00342-2

24. Orii T, Hirasawa M, Seto T (2003) Tunable, narrow-band light emission from size-selected Si nanoparticles produced by pulsed-laser ablation. Appl Phys Lett 83:3395-3397. https:// doi.org/10.1063/1.1621457

25. Lung J-K, Huang J-C, Tien D-C, Liao C-Y, Tseng K-H, Tsung T-T, Kao W-S, Tsai T-H, Jwo C-S, Lin H-M, Stobinski L (2007) Preparation of gold nanoparticles by arc discharge in water. J Alloy Compd 434-435:655-658. https://doi.org/10.1016/j.jallcom.2006.08.213

26. Lo C-H, Tsung T-T, Lin H-M (2007) Preparation of silver nanofluid by the submerged arc nanoparticle synthesis system (SANSS). J Alloy Compd 434-435:659-662. https://doi.org/10.1016/j.jallc om.2006.08.217

27. Chen L-C (2010) Preparation of TiO2 nanoparticles by submerged arc nanoparticle synthesis system. J Alloy Compd 495:476-480. https://doi.org/10.1016/j.jallcom.2009.10.215

28. Tsung T-T, Chang H, Chen L-C, Han L-L, Lo C-H, Liu M-K (2003) Development of pressure control technique of an arc-submerged nanoparticle synthesis system (ASNSS) for copper nanoparticle fabrication. Mater Trans 44:1138-1142. https:// doi.org/10.2320/matertrans.44.1138

29. Mansouri SS, Ghader S (2009) Experimental study on effect of different parameters on size and shape of triangular silver nanoparticles prepared by a simple and rapid method in aqueous solution. Arab J Chem 2:47-53. https://doi.org/10.1016/j.arabj c.2009.07.004

30. Salvioni L, Galbiati E, Collico V, Alessio G, Avvakumova S, Corsi F, Tortora P, Prosperi D, Colombo M (2017) Negatively charged silver nanoparticles with potent antibacterial activity and reduced toxicity for pharmaceutical preparations. IJN 12:2517-2530. https://doi.org/10.2147/ijn.s127799

31. Varshneya R (2010) Biogenic synthesis of silver nanocubes and nanorods using sundried stevia rebaudiana leaves. AML 1:232237. https://doi.org/10.5185/amlett.2010.9155

32. Wani IA, Ganguly A, Ahmed J, Ahmad T (2011) Silver nanoparticles: ultrasonic wave assisted synthesis, optical characterization and surface area studies. Mater Lett 65:520-522. https:// doi.org/10.1016/j.matlet.2010.11.003

33. Kasprowicz MJ, Gorczyca A, Janas P (2016) Production of silver nanoparticles using high voltage arc discharge method. CNANO 12:747-753. https://doi.org/10.2174/15734137126661605191 55956

34. Ashkarran AA (2010) A novel method for synthesis of colloidal silver nanoparticles by arc discharge in liquid. Curr Appl Phys 10:1442-1447. https://doi.org/10.1016/j.cap.2010.05.010

35. Zhang H, Zou G, Liu L, Tong H, Li Y, Bai H, Wu A (2016) Synthesis of silver nanoparticles using large-area arc discharge and its application in electronic packaging. J Mater Sci 52:3375-3387. https://doi.org/10.1007/s10853-016-0626-9

36. Mukherji S, Bharti S, Shukla G, Mukherji S (2018) Synthesis and characterization of size- and shape-controlled silver nanoparticles. Phys Sci Rev. https://doi.org/10.1515/psr-2017-0082

Publisher's Note Springer Nature remains neutral with regard to jurisdictional claims in published maps and institutional affiliations. 